\title{
Introduction to the Topical Issue on Laser Dynamics and Nonlinear Photonics
}

\author{
C. Masoller ${ }^{1}$ and I. Brener ${ }^{2}$ \\ 1 Departament de Fisica i Enginyeria Nuclear, Universitat Politecnica de Catalunya, Colom 11, Terrassa 08222, \\ Barcelona, Spain \\ 2 Sandia National Laboratories and Center for Integrated Nanotechnologies, POB 5800, Albuquerque, New Mexico 87185, USA
}

Received 21 April 2010

Published online 12 May 2010 - (C) EDP Sciences, Società Italiana di Fisica, Springer-Verlag 2010

\begin{abstract}
This topical issue highlights some of the latest research in nonlinear dynamics of photonics devices and novel properties of optical materials that can result from their nonlinear response to light. In this introductory article we comment on some of the challenges in the field. We also discuss and summarize the work presented in the contributed articles to this topical issue and its related workshop.
\end{abstract}

Photonics is the science of generation, propagation, manipulation, and detection of light, and has been recently been identified by the European Commission as one of the key enabling technologies (KETs) for Europe [1], i.e., a technology that will be crucial for facing and solving the challenges of the next century. The photonics field has the potential to revolutionize the telecommunications industry by providing low-cost highly efficient and reliable coherent light sources, all-optical switches, etc. Clearly, a good understanding of the role of nonlinearities in lightmatter interactions will help to overcome the limitations of present technology and to design and develop more reliable and efficient photonic devices and materials.

A nonlinear uncontrolled response of an optical medium can result in instabilities that could be potentially detrimental for the operation of a device; however, controlled instabilities can be employed in unconventional applications. A fascinating example is provided by the recent work of Kanter and colleagues [2], who employed a semiconductor laser subject to time-delayed self-feedback in order to develop an ultra-fast random bit generator, which operates reliably at rates up to $300 \mathrm{Gbit} / \mathrm{s}$. The method uses the high derivatives of a chaotic signal, which is the laser intensity digitized at a $20 \mathrm{GHz}$ sampling rate, and that displays picosecond chaotic fluctuations induced by the delayed feedback.

Spatio-temporal optical patterns is another area where one needs a very good understanding of nonlinear effects in light-matter interactions. For example, three-dimensional solitons, also referred to as light bullets, are optical wave packets localized in time and in space with particle-like properties and correspond to solutions that propagate without distortion. They rely on self-focusing nonlinearities that simultaneously balance the effects of dispersion and diffraction. The generation of 3D light bullets is a very challenging task because inherent instabilities can lead to their disintegration. Since the proposal in the 90's [3] a lot of effort has been carried out to try to defy the effects of dispersion and diffraction. Recently, Chong et al. [4] reported the generation of 3D light bullets that propagate linearly without distortion; their method relies on combining Bessel beams in the transverse plane with temporal Airy pulses. The evolution of the 3D light bullets does not critically depend on the material in which they propagate, and are therefore expected to have a broad range of applications.

The constructive use of noise in nonlinear optical systems is another very active topic of research. For instance, a fundamental problem in imaging, where noise can be beneficial, is the detection of low-level or hidden signals. Dylov and Fleischer [5] have recently shown that nonlinear coupling can lead to an exchange of energy by which a weak signal can grow at the expense of noise, hence allowing for noise-assisted image recovery. The authors have demonstrated the phenomenon, referred to as dynamic stochastic resonance, employing as a signal a coherent image of a resolution chart, as a noise source, laser light $(532 \mathrm{~nm})$ passing through a diffuser, and a photorefractive SBN:60 crystal as the nonlinear medium. The results directly imply that adding noise to weak signals can improve transmission fidelity and that nonlinear coupling can be used for coherence filtering and contrast enhancement; the method is therefore particularly interesting for the analysis of signals with ambient backgrounds, such as those occurring in night vision or turbid media.

This topical issue features a selection of articles that were inspired by talks presented at the recent 4th Rio de la Plata Workshop on Laser Dynamics, held in Piriapolis, Uruguay, in December of 2009. In this introductory paper we comment on some of the talks that were presented 
at the workshop and we summarize of the scientific highlights of the articles in this topical issue. We discuss the problem addressed in each article and highlight the main conclusions.

\section{Nonlinear optics}

Wave propagation in periodic nonlinear structures is associated with many exciting possibilities to control, mold and route light for future applications in all-optical information processing. In contrast to light propagation in prefabricated permanent structures, an increased flexibility can be achieved when light itself induces its own periodic structure through the nonlinear response of the material. In the talk, Cornelia Denz (Münster, Germany) presented in Uruguay an overview of several approaches to realize complex one- two- and three-dimensional photonic lattices, as well as complex lattice geometries such as superlattices or 3D crystallographic and quasi-crystallographic lattices [6]. Due to the ability of the photorefractive effect to create refractive index changes at very low laser powers and to realize adaptive and reconfigurable structures, multidimensional, nonlinear, optically induced lattices can be achieved. The interplay between periodicity and nonlinearity has been shown to cause a variety of fascinating nonlinear effects, among them discrete solitons, Zener Tunneling and Bloch oscillations. Denz also discussed nonlinear wave propagation leading to slow light in $1 \mathrm{D}$ phase-engineered lattices, and discrete spatial solitons or clusters of vortices in 2D lattices [7]. Recently, Denz and colleagues [8] investigated experimentally the formation of a reconfigurable 3D nonlinear photonic lattice in a SBN:Ce photorefractive crystal externally biased by a spatially phase-modulated field. The analysis of the generated nonlinear photonic lattice demonstrated the potential of these 3D structures as reconfigurable platforms to investigate nonlinear light-matter interactions in periodic structures.

In photonic crystals strong light confinement due to high-Q resonances and strong electronic confinement in built-in nanostructures such as quantum wells and quantum dots lead to highly-enhanced light-matter interactions. Photonic crystals allow for novel dynamical nonlinear phenomena in which the optical and material time scales are modified with respect to standard semiconductor devices. In his talk, Alejandro Yacomotti (Marcoussis, France) focused on two recent experimental demonstrations concerning time-resolved nonlinear optics in twodimensional photonic crystals, that point at nonlinear regimes directly associated either to the increase of the photon lifetime or to the decrease of the thermal dissipation time [9]. Recently Yacomotti et al. demonstrated fast thermo-optical excitability in an InP-based photonic crystal slab close to bistable operation [10]. Bistability can occur in photonic crystals due to the interplay between carrier-induced refractive index change and a low group velocity mode at the $\Gamma$ point of the photonic band structure. An excitable system is known to exhibit an allor-none pulse responses to an external perturbation, de- pending on whether this perturbation is above or below a threshold. For perturbation energies close to the excitable threshold, critical slowing down delays the output pulse for an amount of time comparable to the pulse duration (up to $5 \mathrm{~ns}$ ), which can be potentially useful for the realization of an all-optical delay line [11].

Superresolution microscopy methods are nowadays enabling the resolution of objects that are smaller than the optical diffraction limit. In his talk, Rafael Piestum (Boulder, USA) discussed in particular photoactivationlocalization methods, that work by photoactivating a sparse subset of fluorescent molecules at a given time, and localizing each molecule with great precision. Superresolution images are thus obtained by sequentially photoactivating and localizing different sparse molecule subsets and by combining the position information. New principles extend superresolution to the third dimension by means of point spread function engineering and matched computational reconstruction algorithms. In a recent article Gerke and Piestun [12] presented a three-dimensional scattering approach to the design of aperiodic volume optical elements and explored new functionalities by making use of the available three-dimensional degrees of freedom. Aperiodic volume elements that multiplex spatial and spectral information were numerically designed and experimentally demonstrated.

\section{Quantum optics}

Many fundamental aspects of present photonics rely on statistical aspects of photon-matter interactions. The properties of photon fluctuations are usually assumed to be well described by Gaussian distributions; however, in a number of applications, rare events that cannot be interpreted in terms of Gaussian statistics, occur. In her talk, Martine Chevrollier (Joao Pessoa, Brazil) presented experimental measurements of the elementary step process for photons in a hot vapor and provided evidence of their propagation mode in the vapor as Lévy flights. Recently, Chevrollier and colleagues [13] studied this fundamental aspect of photon statistics by means of observing the anomalous diffusion of photons in hot atomic vapors. The authors measured the step-size distribution of the photons random walk and found that it can be described by a power-law distribution, characteristic of Lévy flights. A further discussion of these observations is presented in an article by the same authors in this topical issue [14].

With possible applications to information storage, quantum computation and communications, the field of quantum optics is generating a large amount of exciting research. Quantum information networks are expected to convey information from one place to another by using entangled light beams. An important step towards quantum entanglement was presented by Paulo Nussenzveig (Sao Paulo, Brazil) in his talk. Nussenzveig and colleagues recently demonstrated experimentally "three-color entanglement" [15], i.e., the generation of entanglement among three beams of light, of different wavelengths $(532 \mathrm{~nm}$, $1062 \mathrm{~nm}$ and $1066 \mathrm{~nm}$ ). Their method relied on the use 
of an optical parametric oscillator (OPO), consisting of a nonlinear crystal placed inside a cavity. Light that is incident on the crystal undergoes parametric downconversion, a nonlinear process whereby an incident photon is converted into a pair of longer-wavelength photons. This technique opens potential applications for quantum information networks, since the OPO pump field at $780 \mathrm{~nm}$ can interact resonantly with $\mathrm{Rb}$ atoms and the information stored in the atomic sample can be transferred to a telecom wavelength $(1560 \mathrm{~nm})$ suitable for propagation through a low-loss optical fiber. The properties of light fluctuations resulting from resonant interaction with an atomic system were discussed by Arturo Lezama (Montevideo, Uruguay), who described in his talk recent experiments showing significant light squeezing after single passage of light through an atomic vapor, and presented a theoretical interpretation of the observed phenomenon.

In spite of all the exciting developments being carried out in the field of quantum optics, some fundamental issues related to basic principles of quantum mechanics remain unsolved. The Einstein-Podolsky-Rosen (EPR) paradox is one of the most enduring and challenging problems in the history of Physics. It implies a contradiction between Quantum Mechanics and basic notions on the locality of natural phenomena. The so-called "non ergodic theories" reconcile Quantum Mechanics with the intuitive local view of the world by hypothesizing that randomness at the quantum level is actually the consequence of some complex (eventually chaotic) underlying dynamics. While these dynamics can not be detected by measuring stationary probabilities, they may be revealed through time series analysis. Specifically, through the analysis of the series formed by the time of observation of each single event (the detection of a photon). In his talk Alejandro Hnilo (Villa Martelli, Argentina) discussed a recent work done in his group [16], where they analyzed the raw data of the Innsbruck's experiment, performed more than ten years ago under the hypothesis of strict Einstein locality, as well as data that Hnilo and coworkers obtained after building their own time stamping setup. They used the two complementary data sets to disprove a class of non-ergodic theories that could not be disproved by experiments that measure average rates only, and that had remained untested until now.

\section{Dynamics of lasers and applications}

In the area of nonlinear laser dynamics, several talks highlighted some of the recent developments in the field.

The field of laser synchronization is still very active and attracts a lot of attention. In his talk, Jose Rios Leite (Recife, Brasil) discussed the time leadership competition in the synchronized excitable behavior of two coupled chaotic semiconductor lasers. Experiments recently done by Martinez Avila and Rios Leite [17] showed synchronized excitable low-frequency fluctuations (LFFs) with isochronous, time leading, or time lagging behavior whose stability was shown to depend on a simple relation between the feedback delay time and the mutual coupling delay time. The dynamics may have one of the lasers with a fixed time leadership or have its leading time switching values by discrete steps depending on the values of the coupling and feedback times. Nearly symmetrical lasers can also have intermittent time leadership exchange always maintaining the excitable LFF spikes synchronized in the long time scale. Coupled asymmetrical lasers also follow the conditions for the allowed lag-times between events. Numerical calculations with rate equations for monomode optically coupled semiconductor lasers verify the predicted stability conditions for synchronization. Synchronism with intermittent time leadership exchange was also observed. The selection condition for the lag-times appears to be generic in mutually delay-coupled dynamical systems and has applications in schemes of encrypted communications.

The potential application of synchronized optical chaos to secure communication systems was discussed by Laurent Larger and Maxime Jacquot (Besançon, France). Recently, Larger, Jacquot and coworkers [18] demonstrated that fast, reliable, highly complex, and controllable chaos can be generated by a novel electro-optic nonlinear delay setup, that relies on combining optical phase modulation, differential phase shift keying demodulators, and the dynamical principles of the Ikeda ring cavity [19]. Broadband phase-chaos synchronization was demonstrated employing an emitter-receiver pair of the setup. The capability of $10 \mathrm{~Gb} / \mathrm{s}$ transmission over an installed metropolitan network of more than $100 \mathrm{~km}$ demonstrates the application of the setup for the realization of novel chaos-based secure communication systems.

Another way to generate synchronized chaos, by coupling two semiconductor lasers through their nonlasing orthogonal polarization mode, was discussed by Athanasios Gavrielides (Air Force Research Laboratory, USA). In his talk, he presented a detailed theoretical and experimental analysis of the emission dynamics of two lasers coupled in such configuration. Recently, Gavrielides and colleagues [20] observed experimentally metastable asymmetric wave squares and presented an analytical model that captures the essential features of the metastable waveforms, showing analytically that they approach and vanish exponentially to the steady states for long delays. Their results indicate that the metastable states are indeed sustained by the noise that is intrinsically present in diode lasers, and therefore provide a theoretical interpretation for the experimentally observed waveforms.

Another example of the effect of noise, in transforming a transient dynamics into a noise-sustained one, was presented by Cristina Masoller (Terrassa, Spain), who discussed in her talk the dynamics of a semiconductor laser with delayed optical feedback, focusing on the interplay between noise and delay-induced multistability. The delayed feedback renders the laser multistable, with a set of coexisting fixed points, and induces low-frequency fluctuations (LFFs), i.e., sudden intensity dropouts at irregular times. Within the framework of the deterministic (noise-free) Lang-Kobayashi model [21], for a large 
range of realistic laser parameters the LFFs are just a transient dynamics towards a stable fixed point. Masoller and colleagues recently analyzed the statistical properties of the transient LFFs [22] and investigated the influence of various parameters, finding that realistic values of the noise strength do not affect the average transient time or its distribution, but on the other hand, nonlinear gain saturation was found to have a strong influence, by increasing both, the duration of the LFF transients and the probability of noise-induced escapes from the stable fixed point. Their results therefore provide a theoretical interpretation for the sustained LFFs observed experimentally, which can be interpreted as due to the interplay of noise and various nonlinear light-matter interactions, which are phenomenologically represented in the Lang-Kobayashi model by a gain saturation coefficient.

The following articles in this topical issue deal with various aspects of nonlinear dynamics of lasers and applications.

In [23] Lüdge and Schöll study the nonlinear dynamics of quantum-dot lasers, focusing on the turn-on and the resulting relaxation oscillation features. The authors use a microscopic rate-equation model which separately treats the dynamics of electrons and holes. They discuss the influence of wetting layer doping and show that the inclusion of separate dynamics of holes and electrons is crucial for explaining the dynamic behavior of a quantum dot laser with a doped wetting layer. Introducing p-doping drastically reduces the damping of the turn-on relaxation oscillations, which is a significant feature influencing the modulation response; on the other hand, $n$-doping is found to increase the damping of the turn-on relaxation oscillations.

In [24] Kelleher et al. describe an experimental method for determining the relative phase of an injected laser with respect to the master injection laser. The technique is successfully tested on an injected quantum well laser. The experimentally constructed phaser plots are compared to numerically constructed ones, showing an excellent agreement. An extension of the technique is proposed in order to recover not only the electric field information, but also the gain dynamics. The method by which full 3D dynamics can be recorded experimentally is described and applied to an injected quantum dot laser.

In [25] Kovanis et al. present a detailed investigation of the phase space of injection-locked semiconductor lasers. Lyapunov phase diagrams show the distribution of stable, periodic and chaotic laser output across wide ranges of injection intensities and for both, positive and negative detuning. These diagrams complement earlier results by describing how steady states and periodic modes are organized in parameter space and demonstrate the rich variety of dynamic regimes exhibited by injection-locked semiconductor lasers.

In [26] Meucci and coworkers study experimentally and theoretically the dynamics of a semiconductor laser with AC-coupled nonlinear optoelectronic feedback. Periodic and chaotic attractors displaying excitable features are reported, which allow the authors to extend the fixed point based excitability concept to the case of higherdimensional attractors. The results also allow for the interpretation of the chaotic spiking regime in terms of excitability of a chaotic attractor, where the small chaotic background spontaneously triggers large excitable spikes in an erratic but deterministic sequence.

In [27] Masoller and Oria present a theoretical and experimental study of the frequency dynamics of semiconductor lasers, considering two specific configurations: (i) a conventional diode laser subjected to orthogonal, polarization-rotated optical feedback from an external cavity, such that the feedback light is spectrally filtered an atomic vapor placed in the external cavity; (ii) an extended-cavity diode laser with an intracavity stronglysaturated resonant vapor. In both configurations a variety of dynamic frequency regimes are observed, including frequency bistability, frequency locking to an atomic resonance, and frequency irregular instabilities, which are accompanied by an almost constant output power, i.e., the dynamical behavior occurs mainly in the frequency space, leaving largely unperturbed the laser intensity. They develop theoretical models describing the frequency dynamics in both configurations, and the results obtained through numerical calculations are found to be consistent with the observed frequency dynamics at a nearly constant, stable output power. They include results for a dispersive external-cavity filter that reproduce well previous observations.

In [28] Gelens et al. study experimentally and theoretically the non-linear dynamical behavior of semiconductor ring lasers with imperfect Z2 symmetry. Bifurcation analysis is performed on the basis of rate equations as well as a reduced $2 \mathrm{D}$-model allowing to capture the main dynamical features arising from the competition between the two counter-propagating modes of the ring. Different dynamical regimes were experimentally studied focusing on multistable and excitable behaviors and a lot of care has been taken for the description of the different dynamical scenarios.

In [29] Kofler et al. describe in detail the development of a miniature $\mathrm{Nd}+\mathrm{Cr}$ :YAG laser to be used, among other possible applications, as a spark plug in internal combustion motor engines. Practical details are presented and discussed. It is shown that stable operation with nanosecond 1064-nm emitted pulses of energy up to $15 \mathrm{~mJ}$ under $600 \mathrm{~W}$ pumping can be achieved.

\section{Cavity Solitons and Patterns}

In [30] Amiranashvili et al. derive a simple non-envelope model for pulses propagating in time within a transparency window of a nonlinear dispersive medium with an instantaneous cubic nonlinearity. For this model they investigate integrals of motion and demonstrate that a uniformly moving non-envelope soliton does not exist. The only possible localized solution is the solitary breather with some intrinsic dynamics in the comoving frame. Classical envelope solitons oscillating in the comoving frame appear for a longer pulse for which the model is equivalent 
to the standard nonlinear Schrödinger equation. While the model requires a low-order partial differential equations (i.e., containing only lower-order derivatives) and thus restricts the number of parameters that can be effectively related to the optical medium, it has the advantage of allowing analytical analysis of the approximate evolution equations and thus yields qualitative interesting results.

Cavity solitons (CS) are a type of transverse localized structures with properties that make them very attractive for processing and storage information: they can be independently written and erased in the transverse optical field, they are not affected by boundaries and their control can be possible through phase or intensity gradients. In [31] Colombo et al. study the existence of cavity solitons in broad area (large Fresnel number) class A bi-directional ring lasers close to threshold, and thus described by two coupled Ginzburg-Landau equations. They find that pairs of localized solutions referred to as $z$-defects do not qualify as cavity solitons, since the creation of a defect in the transverse plane is unavoidably accompanied by the generation of a phase wave that covers the whole system hereby making the correlation length equal to the system size. Due to this effect, when a second localized structure is created, it is advected by the phase gradient created by the first, hence these localized structures cannot be considered as true cavity solitons. However, the authors also describe two methods for writing several of localized structures: by creating a second before the phase wave of the first reaches the placement of the second, or by injecting an external field of higher frequency.

In [32] Zambrini and Papoff discuss the effect of twopoint nonlocal coupling on the transverse dynamics of passive and active nonlinear optical systems. They focus on nonlocality that is induced through a displaced feedback. A detailed study of convective and absolute instabilities and their consequences on chirping and splitting of localized perturbations is presented, extending their recent studies [33] to a two-dimensional space. The effects of noise (for example, noise sustained structures) are analyzed within the framework of a set of equations valid for a class A laser. Modification of the model parameters allows for a wide variety of behaviors and the possibility to independently tune phase and group velocities. Some specially interesting results are the possibility to operate a laser with displaced feedback as a beam splitter and the profiles obtained through numerical integration in two dimensions showing some unusual behaviors that are interesting from an experimental point of view.

\section{Metamaterials and plasmonics}

In the area of metamaterials and plasmonics, the Uruguay Workshop had a number of presentations both theoretical and experimental, that highlighted some of the latest developments in this fast paced area.

The ability to tailor the permittivity and permeability of a media at length scales much smaller than the wavelength of light, has enabled a number of astonishing predictions that could someday change the way we design and fabricate optical elements. Examples of these are negative refraction, superlensing, cloaking, etc.

A fundamental issue related to metamaterials is the arguments made by Landau and Lifshitz [34] in which the magnetization (and thus, the permeability), loses its meaning at relatively low frequencies. This problem was addressed by Roberto Merlin (Ann Arbor, USA) in his talk, who recently demonstrated [35] that the Landau and Lifshitz arguments do not apply to composites made of substances with $\operatorname{Im} \sqrt{\epsilon_{s}} \gg \lambda / l$ or $\operatorname{Re} \sqrt{\epsilon_{s}} \sim \lambda / l$, where $\epsilon_{s}$ and $l$ are the complex permittivity and the characteristic length of the particles respectively, and $\lambda \gg l$ is the vacuum wavelength. The double constraint $\operatorname{Im} \sqrt{\epsilon_{s}} \gg \lambda / l \gg 1$ poses severe limitations for observing magnetism at arbitrarily high frequencies. Because they have a large extinction coefficient, metals are to be favored at optical frequencies, and the measured values of the permittivity of noble metals indicate that magnetic behavior can persist at frequencies up to $1.510^{14} \mathrm{~Hz}(\lambda \sim 2.5 \mathrm{~nm})$.

In [36] O'Hara et al. present a model based on the transfer matrix approach to determine effective medium properties of meta-films. The model provides an accurate, unique, and rapid method to determine the effective parameters of metamaterials. Of critical importance is that the model properly accounts for the integrated effects of metafilms and their bounding media, thus offering a great accuracy in practical metamaterial design. Also, once the single-layer response of a metafilm is determined, the model allows for calculating the response of any number of layers. Measurements and examples prove the model validity and reveal practical considerations in the design of metamaterial devices, such as gradient-index lenses.

A great challenge in nowadays nanoscale technology arises from quantum vacuum dispersive forces, generally known as Casimir forces. Such forces are responsible for "stiction" in nano-machines and could be used for contactless force transmission. The holy grail in the field is to achieve Casimir repulsion (also known as "quantum levitation") between air-separated surfaces. In his talk Diego Dalvit (Los Alamos, USA) discussed recent proposals of using different types of metamaterials, including magnetic, polaritonic, plasmonic, and chiral, to achieve Casimir repulsion. Recently, Dalvit and coworkers [37] described theoretically the changes that occur in the Casimir force between two surfaces when one or both surfaces are replaced with a metamaterial medium with magnetic permeability. They analyzed the possibility of a repulsive Casimir force and discussed the reduction in attraction as a function of the strength of the magnetic activity compared to the permeability of the constituent materials. They found that Casimir repulsion decreases with magnetic dissipation, and the sign of the force depends sensitively on the degree of optical anisotropy of the metamaterial and on the form of the frequency dependency of the magnetic response.

Another relevant issue in surface interaction at the nanoscale is heat transfer. While it is usually assumed that the radiative heat transfer between two parallel surfaces has an upper bound given by the blackbody radiation, 
at the nanoscale this can be enhanced by several orders of magnitude, specially when surface phonon polaritons can be excited. In his talk, Jean-Jacques Greffet (Paris, France) discussed the contribution of surface phonon polaritons to radiative heat transfer at the nanoscale, and presented a detailed quantitative comparison between theory and experiments for a sphere and a plate. An experimental set-up recently employed by Greffet and co-workers [38] allows measuring the conductance for plate-sphere gaps varying between $30 \mathrm{~nm}$ and $2.5 \mu \mathrm{m}$. The concepts of black body emission are valid in the limit far field (when the distance between the two surfaces is much larger than the characteristic wavelength of thermal radiation), and they showed how thermal radiation can become monochromatic and coherent when measured in the near field (then the distance between two surfaces is comparable to the characteristic wavelength of thermal radiation) in the presence of surface phonon polaritons. These effects could have far-reaching implications as they pave the way to the design of submicron nanoscale heaters that could be used for heat-assisted magnetic recording, heat-assisted lithography and thermophotovoltaics.

Dealing with the same wavelength range (the thermal infrared) Igal Brener (Sandia, USA) presented a talk on the latest results obtained by the Sandia team on the fabrication of 3D infrared metamaterials using a novel technique called "membrane projection lithography" [39]. This technique enables the fabrication of true out of plane metamaterial resonators with sub-micron resolution and the extension to multilayer structures. His group recently demonstrated split-ring resonators evaporated on the facets of submicron cubes and on curved partial spheres. This technique can be used multiple times in order to create thick, 3D stacks, and the metallic resonators evaporated on the walls retain the nanometerscale dimensions required for metamaterials at these frequencies.

Composites (photonic glasses and colloids) containing metallic nanoparticles display enhanced nonlinear susceptibilities and increased luminescence efficiencies due to the influence of surface plasmons. The optical response of these materials can be controlled by changing the volume fraction occupied by the nanoparticles and their physical characteristics. In his talk, Cid de Araujo (Recife, Brazil) presented a review of recent activity done in Recife on nonlinear optical spectroscopy of composites containing metallic nanoparticles. Recently, they measured [40] the nonlinear refractive index of a NaPO3-WO3-Bi2O3 glass with different relative amounts of the constituents and reported large values of the nonlinear refractive index accompanied by very small nonlinear absorption. These observations demonstrate that these materials can have potential applications in photonic devices such as all-optical switches.

The interface between materials with different signs of their permittivities or permeabilities supports electromagnetic surface waves, also known as surface plasmon polaritons, these are waves which are trapped at the interface and whose electromagnetic fields decay exponentially into both media. In [41] Cuevas and Depine study theoretical the electromagnetic field distribution associated with such surface plasmon polaritons, at a sinusoidally corrugated interface between a conventional dielectric medium and a metamaterial. They employ a perturbative method, valid in the limit of small groove depth to wavelength ratio, that drastically reduces the computational cost and that was recently employed by the authors [42] to calculate the radiation characteristics of electromagnetic eigenmodes at the corrugated interface of ideally transparent left-handed materials. Interesting results showing the effects of a shallow sinusoidal corrugation form of the interface are presented and compared to the results of the flat interface. Unusual phenomena produced by the presence of a metamaterial in one side of the interface are discussed.

In [43] Mártin and Hoyuelos study a generalization of the Lugiato-Lefever equations $[44,45]$, to the case in which the nonlinear medium is a metamaterial. The authors consider the spatio-temporal dynamics in a plane transverse to light propagation, in a ring cavity with plane mirrors filled with a negative index material. The material possesses both, Kerr magnetic nonlinearity, and conventional electric Kerr nonlinearity, and also, small dissipation. The model predicts that the states produced in a metamaterial with negative refraction index will also exist in a material with a positive refraction index as long as the signs of the rest of the model parameters are inverted. In [43] the authors extend their previous analysis [46] to the vectorial case, taking into account the polarization degree of freedom of the electric and magnetic field amplitudes.

In [47], Alexander Popov discusses some extraordinary properties of coherent nonlinear-optical coupling of ordinary and backward electromagnetic waves in negativeindex metamaterials, such as second-harmonic generation, difference-frequency generation and optical parametric amplification. Numerical simulations show the feasibility of compensation of strong losses inherent to nanostructured negative-index plasmonic metal-dielectric composites. All-optical tailoring of transparency and reflectivity is shown to be possible through coherent nonlinear-optical energy transfer from the ordinary control to the negativeindex, backward-wave field.

We conclude the introductory article to this topical issue by thanking not only the authors, who made a special effort in writing high-level papers with general introductions but also the reviewers, who provided invaluable help in critically evaluating the papers that now form the present topical issue. We are also most thankful to Jorge Tredicce, for suggesting the publication of this theme issue, and to Solange Guéhot in the Editorial Office of EPJD, for her invaluable support and excellent technical assistance in making it possible.

\section{References}

1. EU press release IP/09/1394

2. I. Kanter, Y. Aviad, I. Reidler, E. Cohen, M. Rosenbluh, Nat. Photon. 4, 58 (2010)

3. Y. Silberberg, Opt. Lett. 15, 1282-1284 (1990) 
4. A. Chong, W.H. Renninger, D.N. Christodoulides, F.W. Wise, Nat. Photon. 4, 103 (2010)

5. D.V. Dylov, J.W. Fleischer, Nat. Photon. (2010) DOI: 10.1038/NPHOTON. 2010.31

6. J. Xavier, M. Boguslawski, P. Rose, J. Joseph, C. Denz, Adv. Mater. 22, 356 (2010)

7. B. Terhalle, D. Gories, T. Richter, P. Rose, A.S. Desyatnikov, F. Kaiser, C. Denz, Opt. Lett. 35, 604 (2010)

8. X. Xavier, P. Rose, B. Terhalle, J. Joseph, C. Denz, Opt. Lett. 34, 2625 (2009)

9. A. Yacomotti, F. Raineri, C. Cojocaru, P. Monnier, J.A. Levenson, R. Raj, Phys. Rev. Lett. 96, 093901 (2006)

10. A. Yacomotti, P. Monnier, F. Raineri, B. Ben Bakir, C. Seassal, R. Raj, J.A. Levenson, Phys. Rev. Lett. 97, 143904 (2006)

11. M. Brunstein, R. Braive, R. Hostein, A. Beveratos, I. Rober-Philip, I. Sagnes, T.J. Karle, A.M. Yacomotti, J.A. Levenson, V. Moreau, G. Tessier, Y. De Wilde, Opt. Express 17, 17118 (2009)

12. T.D. Gerke, R. Piestun, Nat. Photon. 4, 188, 2010

13. N. Mercadier, W. Guerin, M. Chevrollier, R. Kaiser, Nature Phys. 5, 602 (2009)

14. M. Chevrollier, N. Mercadier, W. Guerin, R. Kaiser, Eur. Phys. J. D 58, 161 (2010)

15. A.S. Coelho, F.A.S. Barbosa, K.N. Cassemiro, A.S. Villar, M. Martinelli, P. Nussenzveig, Science 326, 823 (2009)

16. M.B. Agëro, A.A. Hnilo, M.G. Kovalsky, M.A. Larotonda, Eur. Phys. J. D 55, 705 (2009)

17. J.F. Martinez Avila, J.R. Rios Leite, Opt. Express 17, $21442(2009)$

18. R. Lavrov, M. Peil, M. Jacquot, L. Larger, V. Udaltsov, J. Dudley, Phys. Rev. E 80, 026207 (2009)

19. K. Ikeda, K. Kondo, O. Akimoto, Phys. Rev. Lett. 49, $1467(1982)$

20. D.W. Sukow, A. Gavrielides, T. Erneux, B. Mooneyham, K. Lee, J. McKay, J. Davis, Phys. Rev. E 5, 025206(R) (2010)

21. R. Lang, K. Kobayashi, IEEE J. Quantum Electron. 16, $347(1980)$

22. J. Zamora-Munt, C. Masoller, J. García-Ojalvo, Phys. Rev. A 81, 033820 (2010)

23. K. Lüdge, E. Schöll, Eur. Phys. J. D 58, 167 (2010)
24. B. Kelleher, D. Goulding, B. Baselga Pascual, S.P. Hegarty, G. Huyet, Eur. Phys. J. D 58, 175 (2010)

25. V. Kovanis, A. Gavrielides, J.A.C. Gallas, Eur. Phys. J. D 58, $181(2010)$

26. K. Al-Naimee, F. Marino, M. Ciszak, S.F. Abdalah, R. Meucci, F.T. Arecchi, Eur. Phys. J. D 58, 187 (2010)

27. C. Masoller, M. Oria, Eur. Phys. J. D 58, 191 (2010)

28. L. Gelens, S. Beri, G. Van der Sande, G. Verschaffelt, J. Danckaert, Eur. Phys. J. D 58, 197 (2010)

29. H. Kofler, E. Schwarz, E. Wintner, Eur. Phys. J. D 58, 209 (2010)

30. Sh. Amiranashvili, A.G. Vladimirov, U. Bandelow, Eur. Phys. J. D 58, 219 (2010)

31. L. Colombo, L. Gil, J.R. Tredicce, Eur. Phys. J. D 58, 227 (2010)

32. R. Zambrini, F. Papoff, Eur. Phys. J. D 58, 235 (2010)

33. F. Papoff, R. Zambrini, Phys. Rev. A 79, 033811 (2009)

34. L. D. Landau, E.M. Lifshitz Electrodynamics of Continuous Media (Pergamon Press, Oxford, 1960), p. 251

35. R. Merlin, Proc. Natl. Acad. Sci. USA 106, 1693 (2009)

36. J. O'Hara, A. Azad, A. Taylor, Eur. Phys. J. D 58, 243 (2010)

37. F.S.S. Rosa, D.A.R. Dalvit, P.W. Milonni, Phys. Rev. Lett. 100, 183602 (2008)

38. E. Rousseau, A. Siria, G. Jourdan, S. Volz, F. Comin, J. Chevrier, J.J. Greffet, Nat. Photon. 3, 514 (2009)

39. D.B. Burckel, P. Davids, I. Brener, G.A. Ten Eyck, A.R. Ellis, J.R. Wendt, B.S. Passmore, E.A. Shaner, M.B. Sinclair, Proc. SPIE - Int. Soc. Opt. Eng. 7392, 739204 (2009)

40. F.E.P. dos Santos et al., J. Appl. Phys. 106, 063507 (2009)

41. M. Cuevas, R.A. Depine, Eur. Phys. J. D 58, 249 (2010)

42. M. Cuevas, R.A. Depine, Phys. Rev. Lett. 103, 097401 (2009)

43. D.A.Mártin, M. Hoyuelos, Eur. Phys. J. D 58, 257 (2010)

44. L.A. Lugiato, R. Lefever, Phys. Rev. Lett. 58, 2209 (1987)

45. L.A. Lugiato, W. Kaige, N.B. Abraham, Phys. Rev. A 49, 2049 (1994)

46. D.A. Martin, M. Hoyuelos, Phys. Rev. E 80, 056601 (2009)

47. A.K. Popov, Eur. Phys. J. D 58, 263 (2010) 